\title{
Directional enrichment of directly cloned PCR products
}

\author{
David Horn \\ London School of Hygiene \& Tropical Medicine, London, UK
}

BioTechniques 39:40-46(July 2005)

TA cloning is one of the simplest and most efficient methods for the direct cloning of PCR products. However, it is often desirable to isolate these products cloned in a specific orientation, and strategies of varying complexity and cost are available to facilitate directional cloning. Here I report a novel approach that combines TA cloning with directional enrichment and blue-white color screening. The vector is modified for directional enrichment using Eam1105I, PacI, and blue-white (called DEEP ${ }^{\text {blue }}$ ) cloning by integrating a $L a c Z$ cassette flanked by asymmetrical Eam $1105 \mathrm{I}$ recognition sequences at the cloning site. For DEEP blue cloning, one PCR primer must have six additional bases (AATTAA) at the $5^{\prime}$ end. The restriction enzyme Eam1105I from Fermentas (Sunderland, UK) or the isoschizomer EclHKI from Promega (Southampton, UK) and PacI from New England Biolabs (Herts, UK) are used to generate the T-ends on the vector and to enrich the desired recombinants, respectively. Almost any vector can be modified for DEEP ${ }^{\text {blue }}$ cloning and I demonstrate rapid and efficient directional enrichment using this strategy. This method provides a cost-effective (Eam1105I and PacI cost less than $\$ 1.25$ per reaction) option for high-throughput or even routine cloning.

In most molecular biology laboratories, plasmid construction remains a costly and time-consuming exercise. With so much genome sequence data available, more recombinant vectors are being produced than ever before, often sets of related constructs containing different sequences repre- senting a panel of particularly interesting genes. It is therefore highly desirable to develop tools that alleviate the plasmid construction bottleneck. Although several commercially available vectors allow efficient highthroughput cloning, the selection of vectors is limited, and many rely on steps with which many researchers have no first-hand experience. Only simple, versatile technologies that employ familiar procedures are likely to be widely tested and adopted by the research community. Accordingly, I have devised a novel method that is labor-saving, cost-effective, does not suffer problems caused by end-sensitivity of restriction enzymes, and minimizes problems with internal restriction sites within the amplified sequence.

TA cloning exploits the nontemplate-dependent terminal transferase activity of certain thermophilic DNA polymerases, including Thermus aquaticus (Taq) DNA polymerase (Fermentas). The activity preferentially adds a single adenosine to the $3^{\prime}$ ends of double-stranded DNA to produce $3^{\prime}$ A overhangs. Complementarity with $3^{\prime} \mathrm{T}$ overhangs on a linear vector allows for efficient PCR product-vector ligation. When the aim is to express recombinant proteins with fluorescent or epitope tags, it is often necessary to clone PCR products in a specific orientation. Such approaches allow for protein expression monitoring, localization at the cellular and subcellular levels, and protein purification, as well as the analysis of protein dynamics and interactions (1). Directional cloning of PCR products can be achieved using a number of strategies of varying cost and complexity. For example, vector and insert can be ligated following digestion with a pair of restriction enzymes or appropriate hemi-phosphorylation (2). Digestion of the PCR product is not required if abasic sites are engineered into the primers (3). Alternatively, ligation-independent cloning can be achieved using primers with specific approximately 12 nucleotide extensions followed by exonuclease digestion (4).

Previous analysis of TA cloning using vectors linearized with $X \mathrm{cmI}$ to generate $T$ overhangs gave variable results (D. Horn, unpublished results). Eam1105I, however, allowed reproducible, efficient TA cloning (5). To facilitate the isolation of directionally cloned PCR products, the efficiency of the Eam1105I-TA cloning strategy was tested, combined with a strategy designed to eliminate antisense recombinants following TA ligation. pT7-GFP, a vector for the expression of peptides fused to green fluorescent protein [GFP (6)] in Trypanosoma brucei (see homepages.1shtm.ac.uk/ ipmbdhor/dhhome.htm, Resources) was used, but the strategy is compatible with most vectors.

In order to use Eam1105I at the cloning site in combination with ampicillin selection, a single Eam $1105 \mathrm{I}$ site $\left(\mathrm{GACN}_{5} \mathrm{GTC}\right)$ was removed from the $A m p^{\mathrm{r}}$ ( $\beta$-lactamase) gene. This was achieved using EamD1 (5'-CCCGTGGTGTAGATAACTACGATACG-3') and EamD2 (5'-TAGACGTCAGGTGGCACTT- $3^{\prime}$ ) primers and Vent ${ }^{\circledR}$ DNA polymerase (New England Biolabs) to amplify a fragment that was digested with $S s p$ I and used to replace the Eam1105I-SspI fragment from the vector. This altered the $\mathrm{Thr}^{261}$ codon in $A m p^{\mathrm{r}}$ from ACG to ACC and destroyed the Eam1105I site. This $A m p^{r}-E^{\Delta}$ gene was then available for transfer to other vectors on an $S s p \mathrm{I}-A l w \mathrm{NI}$ fragment, an extremely efficient cloning step because the $A m p^{\mathrm{r}}$ gene and the origin of replication are present on separate DNA fragments and are both absolutely required for colony growth on Amp plates.

To facilitate screening for recombinants, a $L a c Z$ cassette consisting of an inducible $l a c$ promoter and a portion of $L a c Z$ that confers $\alpha$-complementation as the stuffer fragment between 
the new Eam1105I sites was chosen. The $L a c Z$ cassette was PCR amplified from pGEM5Zf ${ }^{+}$) (Promega) using the $5^{\prime} \mathrm{Lac}\left(5^{\prime}\right.$-GATCTCTAGACTC$G A G \mathbf{G A C C}$ T TGCGT CCGAGA -
TAGGGTTGAGTGT-3') and $3^{\prime}$ Lac ( 5'-GATCGGATCCGACCGTGCGTCGCGCAACGCAATTAATGTGA$\left.3^{\prime}\right)$ primers. Both primers encode Eam1105I sites (bold above) with a
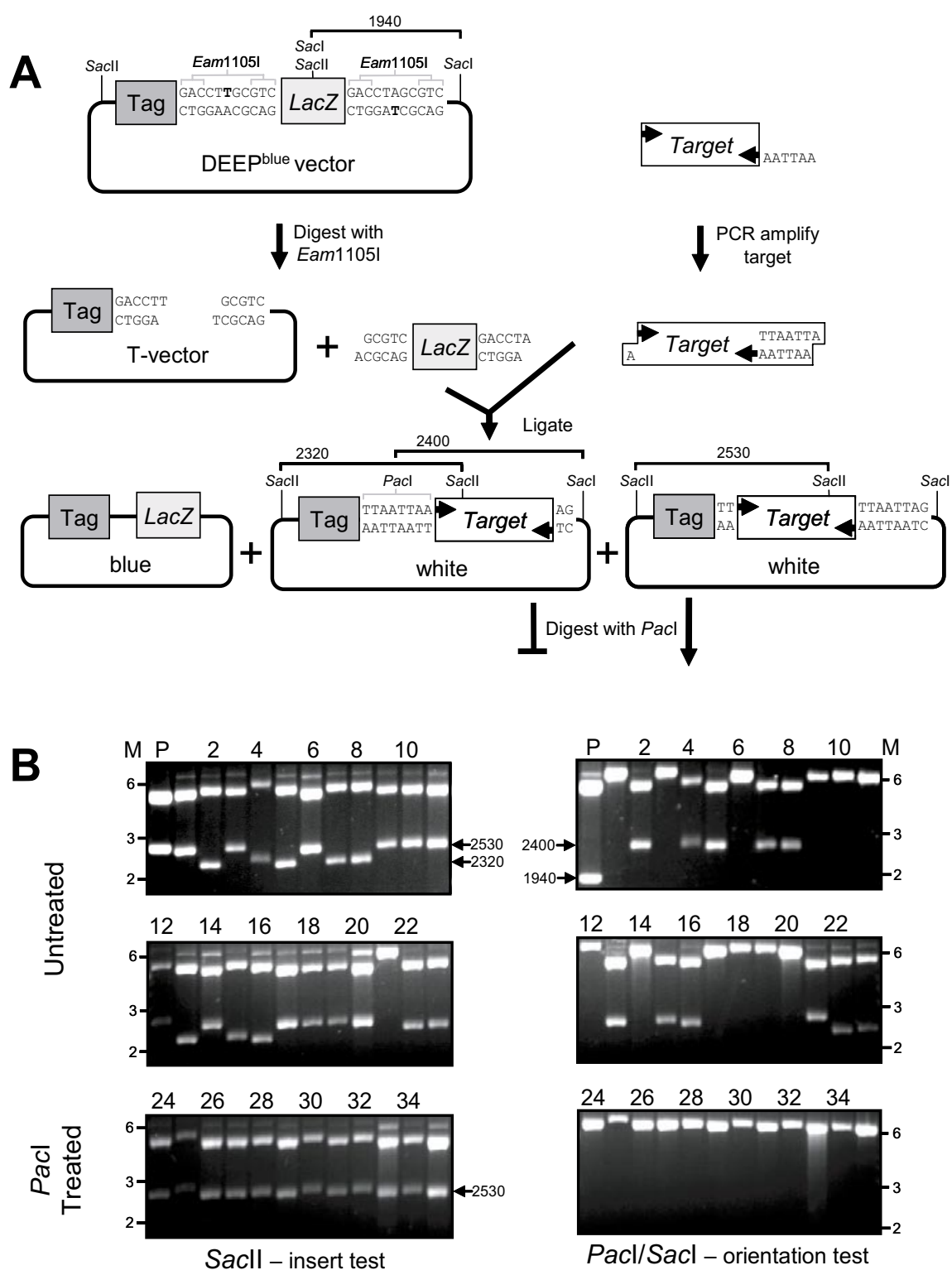

$\mathrm{Pacl} / \mathrm{Sacl}$ - orientation test

Figure 1. Directional enrichment using Eam 1105I, PacI, and blue-white color screening. (A) The flow diagram outlines the procedure. Two micrograms of vector DNA were digested with $10 \mathrm{U}$ Eam 1105I for $1 \mathrm{~h}$ and purified using the MinElute ${ }^{\mathrm{TM}}$ Reaction Cleanup Kit (Qiagen, Valencia, CA, USA). PCR was carried out using Taq DNA polymerase over 30 cycles with Trypanosoma brucei genomic DNA as a template, and PCR products were purified using a QIAquick ${ }^{\circledR}$ PCR Purification Kit (Qiagen). Ligation using T4 DNA ligase (New England Biolabs) was with $10 \%$ of the purified vector and $>10$-fold molar excess of PCR product. The MinElute Reaction Cleanup Kit was used to purify DNA prior to (optional, see text) and following PacI digestion ( $4 \mathrm{U}$ for $1 \mathrm{~h}$ ). All enzymes and kits were used according to the manufacturers' instructions. Relevant restriction sites are indicated. "Tag" is green fluorescent protein (GFP; Reference 6) in this case. (B) Electrocompetent Escherichia coli (DH5 $\alpha$ ) was prepared, transformed, and grown on plates containing isopropyl- $\beta$-D-thiogalactoside (IPTG) and 5-bromo-4-chloro-3indolyl- $\beta$-D-galactopyranoside (X-gal) according to standard protocols (7). White colonies derived from ligation with (lanes 1-23) or without (lanes 24-35) PacI treatment were analyzed. Ten times more white than blue colonies were obtained in both cases. Plasmid DNA was prepared using a single-tube miniprep method (8). P, DEEP ${ }^{\text {blue }}$ parent plasmid; M, GeneRuler ${ }^{\mathrm{TM}} 1 \mathrm{~kb}$ DNA ladder (Fermentas).

central T-residue such that Eam $1105 \mathrm{I}$ cleavage leaves $3^{\prime} \mathrm{T}$ overhangs. The $5^{\prime}$ primer has an additional T-residue (underlined above) that will form part of a $\mathrm{PacI}$ recognition sequence only if the PCR product is ligated in the antisense orientation (Figure 1). The PCR product was digested with $\mathrm{BamHI}$ and $X b a \mathrm{I}$ (italics above) and ligated to similarly digested $\mathrm{pE}^{\Delta}$ vector. $\mathrm{DEEP}^{\text {blue }}$ vector DNA was isolated from a blue colony grown in the presence of isopropyl- $\beta$-D-thiogalactoside (IPTG) and 5-bromo-4-chloro-3indolyl- $\beta$-D-galactopyranoside (X-gal) and checked by restriction enzyme digestion. Retention of the unique BamHI and XhoI sites means that more conventional cloning with blue-white screening remains optional with this vector. Any vector that lacks additional Eam 1105 I sites can be easily modified for DEEPblue cloning using the twostep procedure outlined above.

An open reading frame (ORF) of 675 bp was selected to examine DEEP blue cloning efficiency. Larger inserts should be amenable to this technique, although very large inserts may be more difficult to amplify by PCR and may be unstable when cloned. The primers were BDF25T (5'-AGCAAGAACGAGCGAGATAC- $3^{\prime}$ ) and BDF23T (5'-AATTAACTACGAGCTGTCGTAATCGC- $3^{\prime}$ ). Residues representing the codon immediately following the initiator and the stop codon, respectively, are in bold. Six residues (5'AATTAA-3') immediately adjacent to the stop codon in the Pac-primer (BDF23T) will form part of a PacI recognition sequence (5'-TTAATTAA$\left.3^{\prime}\right)$ only if the PCR product is ligated in the antisense orientation (Figure 1). These recombinant circular plasmids can be linearized by $P a c I$ digestion following ligation. Because PacI requires an 8-bp recognition sequence, it is expected to cut at only one site in every $64 \mathrm{~kb}$ of random DNA sequence and will therefore be absent from most vectors and PCR products.

It is not necessary (or recommended) to purify the $L a c Z$ stuffer away from digested vector because plasmids with reintegrated stuffer are easily identified as blue colonies. As expected, $\mathrm{PacI} / \mathrm{SacI}$ digests of plasmids from white colonies produced a distinct 
pattern when compared to the "parent" (P) vector (Figure 1B, right-hand panels). Without PacI treatment, 22 of 23 white colonies (all except lane 21) from an IPTG/X-gal plate contained plasmid DNA plus insert (Figure $1 \mathrm{~B}$, upper panels), indicating that Eam1105I-TA cloning is very efficient. Of these 22 recombinant plasmids, 10 contained antisense insert (2320-bp fragment following SacII digestion and 2400-bp fragment following PacI/ SacI digestion) and 12 contained sense insert (2530-bp fragment following SacII digestion), which suggests that orientation is random in the absence of PacI. Following PacI treatment, all 12 white colonies analyzed contained plasmid DNA with an insert in the sense orientation (Figure 1B, lower panels). Ligated DNA for Escherichia coli transformation can be derived from primers, template DNA, and uncut vector in a few hours. PacI digestion in $0.5 \times$ ligation buffer worked well (data not shown) so the clean-up step between ligation and PacI digestion (see Figure 1 legend) is probably unnecessary. A potential problem was identified when digested vector was ligated in the absence of PCR product. In this case, white colonies were obtained and found to contain ligated vector that apparently lacked the expected $\mathrm{T}$ overhanging bases. These plasmids were identified and could be linearized by PshAI (Fermentas or New England Biolabs) digestion (target sequence: $\mathrm{GACN}_{4}$ GTC; data not shown). Thus, if $\mathrm{PCR}$ product yield is a concern and the PCR product and vector lack $P$ shAI sites, this enzyme can be used to treat the ligated DNA in parallel with PacI treatment.

The simple DEEPblue strategy allows for efficient, direct cloning of PCR products and isolation of recombinants with inserts in the desired orientation. The technique overcomes problems associated with end sensitivity of restriction enzymes or internal recognition sites (except $P a c \mathrm{I}$ ) within the amplified sequence. The PCR product is ligated directly to the vector that requires no additional treatment beyond restriction enzyme digestion. Unlike many blue-white color screening protocols, the LacZ cassette is completely removed from the desired recombinants. In summary, most vectors are amenable to the DEEP ${ }^{\text {blue }}$ modification procedure for directional enrichment or simply for Eam1105Ifacilitated direct TA cloning. This strategy could be particularly useful for medium- to high-throughput work and for functional genomics.

\section{ACKNOWLEDGMENTS}

I would like to thank Sam Alsford, Martin Taylor, John Kelly, and Brendan Wren for comments on the manuscript. Work in my laboratory is funded by The Wellcome Trust (grant no. 069909).

\section{COMPETING INTERESTS STATEMENT}

The author declares no competing interests.

\section{REFERENCES}

1.Jarvik, J.W and C.A. Telmer. Epitope tagging. 1998. Annu. Rev. Genet. 32:601-618.

2.Zhou, M.Y. and C.E. Gomez-Sanchez. 2000. Universal TA cloning. Curr. Issues Mol. Biol. 2:1-7.

3.Gal, J., R. Schnell, S. Szekeres, and M. Kalman. 1999. Directional cloning of native PCR products with preformed sticky ends (autosticky PCR). Mol. Gen. Genet. 260:569-573.

4.Aslanidis, C. and P.J. de Jong. 1990. Ligation-independent cloning of PCR products (LIC-PCR). Nucleic Acids Res. 18:60696074.

5.Alibu, V.P., L. Storm, S. Haile, C. Clayton, and D. Horn. 2005. A doubly inducible system for RNA interference and rapid RNAi plasmid construction in Trypanosoma brucei. Mol. Biochem. Parasitol. 139:75-82.

6.Chalfie, M., Y. Tu, G. Euskirchen, W.W. Ward, and D.C. Prasher. 1994. Green fluorescent protein as a marker for gene expression. Science 263:802-805.

7.Ausubel, F.M., R. Brent, R.E. Kingston, D.D. Moore, J.G. Seidman, J.A. Smith, and K. Struhl. 1998. Current Protocols in Molecular Biology. John Wiley and Sons, New York.

8.Liu, Z. and N.C. Mishra. 1995. Single-tube method for plasmid miniprep from large numbers of clones for direct screening by size or restriction digestion. BioTechniques 18:214217.

Received 10 January 2005; accepted 11 April 2005.
Address correspondence to David Horn, London School of Hygiene \& Tropical Medicine, Keppel Street, London, WC1E 7HT, UK.e-mail:david.horn@lshtm.ac.uk

To purchase reprints of this article, contact apfeffer@BioTechniques.com 